\title{
Barriers to Research among Faculty at a Health Sciences University
}

\author{
Joseph L Hagan, ${ }^{1, *}$, Paul Armbruster ${ }^{2}$, Richard Ballard ${ }^{2}$ \\ ${ }^{1}$ Baylor College of Medicine, Department of Pediatrics - Neonatology, Houston, Texas, United States \\ ${ }^{2}$ Louisiana State University Health Sciences University, School of Dentistry, New Orleans, Louisiana, United States \\ *Corresponding author: jlhagan@bcm.edu
}

Received November 10, 2018; Revised December 19, 2018; Accepted January 10, 2019

\begin{abstract}
Research in the health sciences is needed in order to continue to improve patient care. A survey was distributed to investigate barriers to research among Dentistry, Nursing and Allied Health Professionals academic faculty at a public health sciences university in the United States. Among the 86 faculty respondents, the majority (58\%) of faculty identified "lack of time for me to do research" to be the single largest barrier to their own research activity and this item had a higher mean score than all other barriers to research examined. Lack of time was significantly more problematic among female faculty $(\mathrm{p}=0.006)$. Research resources were a greater barrier than relevance of research $(\mathrm{p}<0.001)$. Respondents noted that clinical duties are frequently a higher priority than research among health sciences faculty, presumably due to shortages in funding and resources. Given the importance of research to the future quality of patient care, efforts should be made to protect health science faculty members' time and to provide the resources necessary to conduct meaningful research.
\end{abstract}

Keywords: research, academic faculty, nursing, dentistry, allied health

Cite This Article: Joseph L Hagan, Paul Armbruster, and Richard Ballard, "Barriers to Research among Faculty at a Health Sciences University.” American Journal of Educational Research, vol. 7, no. 1 (2019): 44-48. doi: 10.12691/education-7-1-7.

\section{Introduction}

Ongoing high quality research in the health sciences is needed in order to continue to achieve advances in patient care. An estimated $\$ 172$ billion per year is spent on health-related research in the United States [1], but industry invests much more (\$116 billion) than academia (\$12.5 billion). Despite the relatively low level of funding, research productivity is an expectation of most academic faculty in the health sciences. Green [2] estimated that it takes 17 years for an issue to go from a research priority to incorporation into clinical practice, so a reduction in health-related research today will be detrimental to the next generation's healthcare quality. Therefore it is important for health science universities to facilitate research productivity among academic faculty. The purpose of this study was to examine barriers to research among academic faculty at a public university health sciences center in the United States and to investigate how these barriers are related to faculty member characteristics.

\section{Methods}

A cross-sectional survey of faculty at three schools in a United States public university health sciences center was conducted. The schools were Dentistry, Nursing and
Allied Health Professionals (consisting of programs in Audiology, Cardiovascular Sonography, Clinical Laboratory Science, Occupational Therapy, Physical Therapy, Clinical Rehabilitation Counseling, Respiratory Therapy and Speech-Language Pathology). The survey tool was adapted from the Barriers to Nurses' Participation in Research Questionnaire developed and evaluated by Hagan and Walden [3]. As recommended by DeVellis [4], the tool was reviewed by a panel of four senior academic faculty in order to assess appropriateness of the items. At this point, the item "Lack of administrative/clerical support for research grant submission” was added to the "Research Resources" subscale in order to make the tool more appropriate for academic faculty. Additionally, to address the broader target population beyond nurses, the item "Research is not relevant to nursing practice" was changed to "Research is not relevant to clinical practice". So the survey instrument that was administered contained 9 items in the "Research Resources" subscale and 6 items in the "Personal Relevance of Research" subscale, in addition to the independent item "Lack of time for me to do research" which did not load onto either of the two factors. Responses to all items were presented in a 5-point Likert-type scale format with the options, strongly disagree, disagree, neither agree nor disagree, agree, or strongly agree, assigned a score of 1 through 5, respectively, with a higher score indicating a greater extent of agreement that the item represents a barrier to the faculty member's research. 
After approval to conduct this study was obtained from the Institutional Review Board (IRB) the survey was distributed online via Survey Monkey ${ }^{\mathrm{TM}}$ (Palo Alto, CA) in using an email distribution list of all full-time faculty members at the three participating schools. After three weeks a reminder email was sent to encourage participation.

Descriptive statistics for quantitative variables are presented as mean \pm standard deviation (SD). Skewness and kurtosis of responses to individual items were assessed using criteria specified by Muthén \& Kaplan [5]. Chronbach's alpha $(\alpha)$ was used to assess internal consistency of the subscale item responses and interpreted according to the guidelines provided by George and Mallery [6]. The Chi-Square test was used to test for an association between school and response rate. The Paired t-test was used to test for a significant difference in subscale mean scores. The two sample $t$-test for independent samples and Analysis of Variance (ANOVA) were used to compare survey responses among categorical variables having two and more than two groups, respectively, with Tukey's Honestly Significant Differences test used for ANOVA post-hoc analysis. The Pearson Correlation Coefficient (PCC) was used to examine the association of quantitative faculty characteristics with survey responses. For outcomes having more than one significant association, a multiple linear regression model was fit to include as independent variables all faculty characteristics exhibiting a significant bivariate association with the outcome. SAS version 9.4 (SAS Institute Inc., Cary, North Carolina) was used for all statistical analysis. Based on the historical survey response rates of about $20 \%$ at the institution, the estimated 76 survey responses would provide $80 \%$ power to detect a true correlation of $r=0.315$ at the $5 \%$ significance level, which represents a "medium” association according to the criteria of Cohen ([7], p. 79-80).

\section{Results}

Responses were received from 86 of the 381 faculty members to whom the survey was distributed, yielding a response rate of $23 \%$. The response rates for the three participating schools were as follows: School of Nursing $23 / 70=33 \%$, School of Dentistry 34/206 $=17 \%$ and School of Allied Health Professionals 24/105 = 23\%, which represents a statistically significant difference in response rates across schools $\left(X^{2}=8.6, d f=2, p=0.014\right)$. Characteristics of the 86 participating faculty members are summarized in Table 1.

None of the items had a skewness or kurtosis that exceeded 2 in absolute value, the threshold proposed by Muthén and Kaplan [5], so all survey items could be retained without compromising the integrity of the results [8]. For the "Research Resources" subscale $\alpha=0.80$, and for the "Personal Relevance of Research" subscale $\alpha=0.84$, both of which indicate "good" internal consistency [6].

The majority (58\%) of faculty identified "Lack of time for me to do research" to be the single largest barrier to their own research activity, and no other barrier was selected as the largest by more than $10 \%$ of respondents (Table 2). The item "Lack of time for me to do research" also had the highest mean score followed by "Lack of financial or other resources to facilitate research" which was the second most commonly selected largest barrier to research (Table 2). The mean "Research Resources" subscale score $(3.3 \pm 1.7)$ was significantly higher $(t=12.8, d f=85, p<0.001)$ than the mean "Personal Relevance of Research” subscale score $(2.1 \pm 0.8)$.

Table 1. Participating faculty member characteristics

\begin{tabular}{lc}
\hline Characteristic & Frequency* (\%) \\
\hline Gender & \\
Female & 41 / $76(54 \%)$ \\
Male & 35 / $76(46 \%)$ \\
School & \\
Allied Health Professionals & 24 / $81(30 \%)$ \\
Dentistry & 34 / $81(42 \%)$ \\
Nursing & 23 / $81(28 \%)$ \\
Academic Rank & \\
Instructor & 13 / $83(16 \%)$ \\
Assistant Professor & 32 / $83(39 \%)$ \\
Associate Professor & 28 / $83(34 \%)$ \\
Professor & 10 / $83(12 \%)$ \\
Academic Track & \\
Tenure Track & 27 / $74(36 \%)$ \\
Clinical Track & 45 / $74(61 \%)$ \\
Research Track & 2 / $74(3 \%)$ \\
\hline Characteristic & Mean \pm SD (Range) \\
\hline Age in years (n = 68) & $51.9 \pm 10.7(30-75)$ \\
Years Employed at institution (n = 68) & $13.9 \pm 10.0(0.3-39)$ \\
\hline
\end{tabular}

*All characteristics' categories do not have the same total frequency because a different number of responses were missing for each question.

Table 2. Frequencies of selections for the largest barrier to the faculty member's research activity $(\mathrm{n}=\mathbf{8 6})$.

\begin{tabular}{|c|c|c|}
\hline Barrier (Subscale) & $\begin{array}{c}\text { Frequency (\%) Selected as } \\
\text { Largest Barrier }\end{array}$ & $\begin{array}{l}\text { (Rank of Mean Score)* } \\
\text { Mean Score } \pm \text { SD }\end{array}$ \\
\hline Lack of time for me to do research (N/A) & $50(58 \%)$ & (1) $4.1 \pm 1.1$ \\
\hline Lack of financial or other resources to facilitate research (Resources) & $9(10 \%)$ & (2) $3.8 \pm 1.0$ \\
\hline Lack of institutional research infrastructure (Resources) & $6(7 \%)$ & (5) $3.4 \pm 1.2$ \\
\hline Lack of availability of experienced research mentors (Resources) & $5(6 \%)$ & (6) $3.3 \pm 1.2$ \\
\hline Lack of incentive/reward to do research (Resources) & $5(6 \%)$ & (4) $3.5 \pm 1.1$ \\
\hline Lack of research knowledge or skills (Resources) & $4(5 \%)$ & (10) $2.9 \pm 1.2$ \\
\hline I feel intimidated by research (Relevance) & $2(2 \%)$ & (11) $2.5 \pm 1.2$ \\
\hline Research is not part of my job (Relevance) & $2(2 \%)$ & (12) $2.3 \pm 1.1$ \\
\hline I do not have ideas for research project topics (Relevance) & $1(1 \%)$ & (14) $2.1 \pm 1.1$ \\
\hline Lack of leadership support (Resources) & $1(1 \%)$ & (7) $3.2 \pm 1.2$ \\
\hline Lack of research training opportunities (Resources) & $1(1 \%)$ & (8) $3.1 \pm 1.1$ \\
\hline Lack of administrative/clerical support for research grant submission (Resources) & $0(0 \%)$ & (3) $3.6 \pm 1.2$ \\
\hline Lack of accessibility to a Research Committee (Resources) & $0(0 \%)$ & (9) $3.0 \pm 1.2$ \\
\hline My lack of training and educational background (Relevance) & $0(0 \%)$ & (13) $2.1 \pm 1.1$ \\
\hline Research is not very interesting or valuable to me (Relevance) & $0(0 \%)$ & (15) $2.0 \pm 1.0$ \\
\hline Research is not relevant to clinical practice (Relevance) & $0(0 \%)$ & (16) $1.6 \pm 0.8$ \\
\hline
\end{tabular}

*Apparent ties in mean scores were broken by examination beyond the first decimal place. 
Table 3. Association of faculty member characteristics with survey responses

\begin{tabular}{|c|c|c|c|c|}
\hline \multirow[b]{2}{*}{ Characteristic } & \multicolumn{3}{|c|}{ Mean \pm SD* } & \multirow[b]{2}{*}{ Overall Satisfaction } \\
\hline & Research Resources & Relevance of Research & Lack of Time & \\
\hline \multicolumn{5}{|l|}{ Gender } \\
\hline Female & $3.2 \pm 0.9$ & $1.9 \pm 1.0^{\mathrm{B}}$ & $4.3 \pm 1.1^{\mathrm{A}}$ & $2.6 \pm 0.3$ \\
\hline Male & $3.3 \pm 0.8$ & $2.3 \pm 1.0^{\mathrm{A}}$ & $3.7 \pm 1.5^{\mathrm{B}}$ & $2.5 \pm 1.2$ \\
\hline \multicolumn{5}{|l|}{ School } \\
\hline Allied Health Professionals & $3.4 \pm 0.7$ & $2.0 \pm 0.6^{\mathrm{B}}$ & $3.5 \pm 1.5^{\mathrm{B}}$ & $2.5 \pm 1.1$ \\
\hline Dentistry & $3.4 \pm 0.6$ & $2.5 \pm 0.8^{\mathrm{A}}$ & $4.4 \pm 0.6^{\mathrm{A}}$ & $2.6 \pm 0.9$ \\
\hline Nursing & $3.0 \pm 0.7$ & $1.8 \pm 0.7^{\mathrm{B}}$ & $4.4 \pm 0.7^{\mathrm{A}}$ & $2.6 \pm 0.9$ \\
\hline \multicolumn{5}{|l|}{ Academic Track } \\
\hline Tenure Track & $3.2 \pm 0.8$ & $1.9 \pm 0.8$ & $4.2 \pm 0.9$ & $2.4 \pm 0.9^{\mathrm{A}}$ \\
\hline Clinical Track & $3.4 \pm 0.6$ & $2.3 \pm 0.8$ & $4.0 \pm 1.1$ & $2.7 \pm 1.0^{\mathrm{A}}$ \\
\hline Research Track & $4.0 \pm 0.0$ & $1.6 \pm 0.4$ & $4.5 \pm 0.7$ & $1.0 \pm 0.0^{\mathrm{B}}$ \\
\hline \multicolumn{5}{|l|}{ Academic Rank } \\
\hline Instructor & $3.0 \pm 0.9$ & $1.8 \pm 0.7$ & $4.5 \pm 0.5$ & $2.8 \pm 0.8$ \\
\hline Assistant Professor & $3.4 \pm 0.6$ & $2.3 \pm 0.8$ & $4.0 \pm 1.2$ & $2.7 \pm 1.0$ \\
\hline Associate Professor & $3.4 \pm 0.7$ & $2.1 \pm 0.8$ & $4.3 \pm 0.8$ & $2.4 \pm 1.0$ \\
\hline Professor & $3.3 \pm 0.9$ & $1.8 \pm 0.7$ & $3.7 \pm 1.2$ & $2.1 \pm 1.0$ \\
\hline
\end{tabular}

*Groups with different letters are significantly different $(\mathrm{p}<0.05)$.

"Personal Relevance of Research" scores of male faculty $(2.3 \pm 1.0)$ were significantly higher $(t=2.1$, $d f=74, p=0.042)$ than females' $(1.9 \pm 1.0)$. Female faculty $(4.4 \pm 1.1)$ agreed significantly more $(t=2.8$, $d f=74, p=0.006$ ) that "Lack of time for me to do research" hinders research activity than males $(3.7 \pm 1.5)$.

Faculty in the School of Dentistry had significantly higher "Personal Relevance of Research" scores (2.5 \pm 0.8$)$ than faculty in the School of Allied Health Professionals (2.0 $\pm 0.6, p=0.046)$ and School of Nursing (1.8 \pm 0.7 , $p=0.003$ ) (Table 3). The extent of agreement that "Lack of time for me to do research" hinders research activity was significantly lower for faculty in the School of Allied Health Professionals $(3.5 \pm 1.5)$ compared to faculty in the School of Dentistry $(4.4 \pm 0.6, p=0.008)$ and School of Nursing $(4.4 \pm 0.7, p=0.011)$. "Research Resources" scores were marginally higher for School of Dentistry faculty (3.4 \pm 0.6$)$ compared to School of Nursing faculty (3.0 \pm 0.7 ), but the difference did not achieve statistical significance ( $p=0.075)$.

Faculty on the Research Track had significantly lower ( $p=0.043)$ overall satisfaction with opportunities to engage in research $(1.0 \pm 0.0)$ than faculty on the Clinical Track $(2.7 \pm 1.0)$. Faculty on the Clinical Track tended to have higher "Personal Relevance of Research" subscale scores $(2.3 \pm 0.8)$ than faculty on the Research Track (1.6 \pm 0.4$)$, but the difference was not statistically significant $(p=0.073)$.

Age of the faculty member was inversely associated with overall satisfaction with research opportunities ( $r=-0.283, p=0.019)$. Neither time employed at the institution nor academic rank were significantly associated with any of the outcomes ( $p>0.05)$.

After adjusting for gender, faculty in the School of Dentistry had mean "Personal Relevance of Research" scores that were 0.5 points lower than faculty in the other two schools ( $p=0.002)$. After adjusting for academic track, age still exhibited a significant inverse relationship with overall satisfaction with research opportunities ( $p=0.022$ ). For each additional year of age, a faculty member's overall satisfaction with research opportunities score decreased by 0.02 points, on average, after controlling for academic track. For lack of time to do research, there was a significant interaction between gender and school of primary appointment $(p<0.001)$ implying that the relationship between gender and lack of time to do research depends on school of primary appointment and, therefore, cannot be generalized across schools. A stratified analysis found that there was not a significant relationship between gender and lack of time to do research for faculty in the School of Nursing ( $p=0.830$ ) or the School of Dentistry ( $p=0.177)$, but females' mean score for "Lack of time for me to do research" was 1.4 points higher than males in the School of Allied Health Professionals $(p=0.015)$.

\section{Discussion}

This study has identified lack of time to be by far the single largest barrier to research activity. Our finding that lack of time is the largest barrier to faculty research activity is consistent with conclusions drawn from previous studies $[9,10,11,12,13]$ which concluded that time for faculty research was reduced due to a heavy workload. In a survey of universities in the United States, when asked "What are the major barriers to the performance of research at your institution?" more than $80 \%$ of participants' comments indicated lack of time [14]. An obvious solution is to reduce the clinical duties and teaching load of faculty, particularly junior faculty who are trying to build a research track record to strengthen their research credibility. But the reality is that reduced university budgets and faculty-to-student ratios often render this solution impractical.

Lack of time represented a significantly greater barrier to research in the schools of dentistry and nursing where clinical duties compete with research and teaching for faculty members' time. And faculty in the School of Dentistry had significantly higher "Relevance of Research" scores. Together these results could be interpreted as an indication that faculty in the dental school devote more time and effort to clinical duties and they view research activity as a lower priority. This interpretation is 
supported by a dental school faculty member's comment that the "dental school overall seems to have more of a 'clinical' culture and most people couldn't care less about research.” Another dental school faculty member commented that "Being Clinical - it's really hard to find the time to do research between all the teaching and clinical responsibilities”. A third dental school faculty member commented, "It seems that teaching and research are somewhat mutually exclusive for clinical faculty; if we are to be good teachers, carry out our committee responsibilities, and be in clinic $20 \%$ of the week, the result is lack of time and frankly low priority for research."

After "lack of time for me to do research", which represents a stand-alone item, the next 9 items in mean score rank are from the "Research Resources" dimension. Studies in less developed countries [15] have identified financial limitations to be a greater barrier to faculty research than lack of time. Given the recent dramatic budget cuts at the institution where the study was conducted, it would be interesting to do a study to compare more financially stable universities' "Research Resources” subscale scores.

This study found that female faculty generally perceive research to be of greater personal relevance than male faculty, but lack of time is a greater hindrance to their research activity compared to male faculty, particularly in allied health professions. Previous studies have found female faculty to experience more obstacles to research than males [15,16,17]. But a longitudinal study [18] from 2000 to 2010 of medicine, science and engineering academic faculty found that "female faculty published fewer articles and were awarded fewer grants in the baseline period, but their productivity did not differ from male faculty on these measures in subsequent years”. Based on these findings it could be that gender differences are growing smaller over time.

This study observed that overall satisfaction with research opportunities declined with age. One might speculate that younger faculty are more likely to just be happy to have a first job in academia. But no differences were observed with respect to academic rank. Algadheeb and Almeqren [19] found no differences in obstacles to research in terms of faculty member age or academic rank. On the other hand, Karimian et al. [15] found that junior faculty perceived more obstacles to research than senior faculty. Tritton [20] found that Associate Professors had lower research funding levels and publication rates than Assistant Professors and full Professors.

The primary limitation of this study is the low response rate which could potentially cause response bias. However online survey response rates are notoriously low, averaging 30\% (Survey Monkey, 2016), and the response rate in the current study is somewhat higher than other published studies involving surveys related to research $[3,21]$.

This study finds lack of time to do research to be by far the largest barrier to faculty research at a public university health sciences center in the United States. This study's finding that research resources represent a significantly greater barrier to research than personal relevance of research indicates that faculty generally believe research is important to their careers but they lack adequate resources to be productive in research. Given the importance of health-related research to future public health quality, efforts should be made to protect health science faculty members' time and to provide the resources necessary for conducting meaningful research.

\section{Conflicts of Interest}

The authors have no conflicts of interest to report

\section{Data Integrity}

All data presented in this manuscript are real and authentic.

\section{References}

[1] Research America (2017) "US Investments in Medical and Health Research and Development” Accessed 11/23/2018: https://www.researchamerica.org/sites/default/files/RA2017_InvestmentReport.pdf.

[2] Green L.W. (2008) "Making research relevant: It is an evidencebased practice, where is the practice-based evidence" Family Practice, Suppl 1, i20-24.

[3] Hagan J., Walden M. (2015). "Development and evaluation of the Barriers to Nurses' Participation in Research Questionnaire at a large academic pediatric hospital" Clinical Nursing Research, October 2015. pp. 1-19.

[4] DeVellis R.F. (2011). Scale development: theory and applications (3rd ed.). Thousand Oaks, CA: SAGE Publications, Inc.

[5] Muthén B., Kaplan D. (1985). "A comparison of some methodologies for the factor analysis of non-normal Likert variables" British Journal of Mathematical and Statistical Psychology, 38, 171-189.

[6] George D., Mallery P. (2003) SPSS for Windows step by step: a simple guide and reference. 11.0 update (4th ed.). Boston: Allyn \& Bacon.

[7] Cohen, J. (1988). Statistical Power Analysis for the Behavioral Sciences (2nd ed.), Hillsdale, NJ: Lawrence Erlbaum Associates.

[8] Ferguson E., Cox T. (1993). "Exploratory factor analysis: a user's guide" International Journal of Selection and Assessment, 1(2), 84-94.

[9] Parahoo K. (2000) "Barriers to and facilitators of research utilization among nurses in Northern Ireland" Journal of Advanced Nursing, 30, 89-98.

[10] Miller R.D. (2002). "The place of research and the role of academic anaesthetists in anaesthetic departments" Best Practice \& Research. Clinical Anaesthesiology, 16(3), 353-370.

[11] Kuuppelomaki M., Tuomi J. (2003). "Finish nurses' views on their research activities" Journal of Clinical Nursing, 12, 589-600.

[12] Brown JH, Oplatka I. (2005) "Bridging the research-practice gap: barriers and facilitators to research use among school principals from England and Israel" International Journal of Public Sector Management, 18,424-446.

[13] Alghanim S.A., Alhamali R.M. (2011). "Research productivity among faculty members at medical and health schools in Saudi Arabia: Prevalence, obstacles, and associated factors" Saudi Medical Journal, 32(12), 1297-1303.

[14] Doyle, M.P. (2000) Academic Excellence: The Sourcebook, A Study on the Role of Research in the Physical Sciences at Undergraduate Institutions, Tucson, AZ: Research Corporation.

[15] Karimian Z., Sabbaghian Z, Salehi A., Sedghpour B.S. (2012). "Obstacles to undertaking research and their effect on research output: a survey of faculty members' views at Shiraz University of Medical Sciences” Eastern Mediterranean Health Journal, 18(11), 1143-1150.

[16] Andrews N.C. (2002). "The other physician-scientist problem: where have all the young girls gone?” Nature Medicine, 8(9), 439-440.

[17] Guelich J.M., Castro B.H., Rosenberg L.E. (2002). "A gender gap in the next generation of physician-scientists: medical student interest and participation in research" Journal of Investigative Medicine, 50, 412-418. 
[18] Sheridan J., Savoy J.N., Kaatz A., Lee Y-G, Filut A., Carnes M. (2017) "Write more articles, get more grants: The impact of department climate on faculty research productivity" Journal of Women's Health, 26(5): 587-596.

[19] Algadheeb N.A., Almeqren M.A. (2014). "Obstacles to scientific research in light of a number of variables" Journal of International Education Research, 10(2), 101-110.

[20] Tritton T.R., (2002) "The Environment for Scientific Research by Undergraduates: Some Thoughts on Reading the Academic Excellence Study”, Research Corporation Special Report, Tucson, AZ: Research Corporation.
[21] Straka, K. L., Brandt, P., Brytus, J. (2013). "Brief report: Creating a culture of evidence-based practice and nursing research in a pediatric hospital” Journal of Pediatric Nursing, 28, 374-378.

[22] Meybodi F.B., Zarandi, M.M., Hassani M, Rajabi M. (2015). “A survey of barriers to health research promotion” International Journal of Hospital Research, 4(2), 63-70.

[23] Survey Monkey (accessed May 31, 2016) “Smart survey design”. http://s3.amazonaws.com/SurveyMonkeyFiles/SmartSurvey.pdf.

(C) The Author(s) 2019. This article is an open access article distributed under the terms and conditions of the Creative Commons Attribution (CC BY) license (http://creativecommons.org/licenses/by/4.0/). 